Check for updates

Cite this: RSC Adv., 2017, 7, 32806

\title{
Selective filtration of oil/water mixtures with bioinspired porous membranes
}

\author{
Claudia Zeiger, (D) Jana Kumberg, Felix Vüllers, Matthias Worgull, \\ Hendrik Hölscher (iD* and Maryna N. Kavalenka
}

\begin{abstract}
Membranes inspired by special wetting properties of aquatic plant leaves enable selective removal of either oil or water from oil/water mixtures by filtration. Here, we introduce polymeric micro- and nanohaircovered porous membranes fabricated using highly scalable fabrication methods: hot pulling and perforation with microneedles. The as-prepared superhydrophobic/superoleophilic oil-removing membranes are converted into underwater superoleophobic water-removing membranes by argon plasma treatment. Membrane permeability and breakthrough pressures are analyzed and compared to theory, and the efficiency of both types of membranes for oil/water separation is demonstrated.
\end{abstract}

Received 12th May 2017

Accepted 21st June 2017

DOI: 10.1039/c7ra05385a

rsc.li/rsc-advances

materials are suitable for oil/water filtration. ${ }^{6}$ "Oil-removing"

\section{Introduction}

The enormous worldwide consumption of oil and petroleum products leads to increased oil exploration, transportation, storage and processing, which are accompanied by high risk of oil spillage and aquatic pollution. As accidental oil spills negatively impact environment and public health, developing efficient and sustainable methods for cleaning oil spills is of great significance.$^{1-4}$ Existing technologies used for handling oil spills, including chemical dispersion, oil skimmers and in situ burning, have limited efficiency, cause secondary pollution, and are potentially toxic and economically inefficient. ${ }^{2,5}$ Therefore, absorbing oil using selective oil sorbents is a dominant method used in many applications, despite its limited absorption capacity as well as challenging handling and oil recovery. ${ }^{6-8} \mathrm{~A}$ promising alternative oil/water separation technique to absorption is membrane-based filtration. Continuous mixture separation by filtration with high separation capacity and reduced material consumption is widely used in various industries., ${ }^{910}$ Effective oil/water filtration requires special wetting membrane materials with opposite affinities towards water and oil, which allow only one of the liquids to permeate while retaining the other. ${ }^{6}$

Artificial special wetting materials are inspired by natural superhydrophobic surfaces of plant leaves or underwater superoleophobic surfaces of fish scales. ${ }^{11-13}$ Efficient oil/water separation has been recently demonstrated using superhydrophobic leaves of water lettuce Pistia and water fern Salvinia, which oil absorption capacities are higher than common commercial solutions. ${ }^{12}$ In general, two types of special wetting

Karlsruhe Institute of Technology, Institute of Microstructure Technology, Hermann-von-Helmholtz-Platz 1, 76344 Eggenstein-Leopoldshafen, Germany. E-mail: hendrik.hoelscher@kit.edu membranes with a superhydrophobic/superoleophilic surface have high water contact angles (WCA) above $150^{\circ}$ and low oil contact angles (OCA) close to $0^{\circ}$, and separate oil from oil/water mixtures by selective oil permeation. Such wetting behavior occurs from a combination of surface chemistry and surface micro- and nanoscale roughness., ${ }^{\mathbf{5}, 14,15}$ Underwater superoleophobic "water-removing" membranes, on the contrary, separate water by retaining oil while water penetrates through the membrane. Underwater superoleophobicity is a result of water infiltration into the surface structure of the hydrophilic material and, thus, decrease in oil/solid contact area. ${ }^{6,16}$ The selection of the membrane type depends on the application. Oilremoving filters are typically used for oil purification and separation of heavy oil/water mixtures, in which water has higher density compared to oil., ${ }^{6,8,9,16}$ Water-removing membranes are advantageous for light oil/water filtration, wastewater treatment and other water purification processes. Such membranes are characterized by reduced oil/solid contact area, which minimizes the risk of fouling and results in increased membrane lifetime. ${ }^{6,8,16}$

A wide range of oil-removing and water-removing filtration materials has been developed in recent years, based on metallic meshes, textiles, fabrics, polymeric membranes and filter papers. ${ }^{6}$ Methods commonly used to achieve special wetting properties of oil- or water-removing membranes are chemical modification such as oxidization of the membrane material, and deposition of coatings on the membrane surface. ${ }^{3}$ Coating materials include polymers (polytetrafluorethylene, hydrogels), metal compounds $\left(\mathrm{Cu}(\mathrm{OH})_{2}, \mathrm{ZnO}\right.$, nickel stearate), and various nanoparticles., ${ }^{3,17-39}$ In contrast to superhydrophobic oilremoving membranes and underwater superoleophobic waterremoving membranes, membrane materials with switchable wetting properties allow selective separation of either oil or 
water from oil/water mixtures. Stimuli-responsive materials switch their wetting behavior in response to change in external stimuli such as temperature, electric or magnetic field., ${ }^{5,40-43}$ Other membrane materials require prewetting with either oil or water and change their wetting properties in response to the prewetting liquid. ${ }^{16,44-46}$ However, most existing materials can separate only one of the two liquids (oil or water), and development of environmentally friendly porous membranes, which enable selective separation of both liquids and can be fabricated on large scale, still remains a challenge. ${ }^{6}$

Here, we present porous polymeric nanofur membranes, which can separate both oil and water from oil/water mixtures in a continuous filtration process. Nanofur is a polymeric surface covered by a dense layer of random high aspect ratio nano- and microhairs. Its surface topography is inspired by leaves of aquatic plants Pistia and Salvinia. ${ }^{12}$ Polymeric nanofur is fabricated using a scalable hot pulling process, which creates a layer of dense randomly distributed micro- and nanohairs on polymer film surface. ${ }^{47}$ Pores are then perforated in the nanofur films with microneedle stamps or single microneedles. The asprepared porous membrane is superhydrophobic/ superoleophilic and allows oil to penetrate through pores while retaining water, thus, efficiently removing oil from the mixture in filtration process. Additionally, oil-removing porous nanofur is converted into an underwater superoleophobic water-removing filter by plasma treatment, resulting in removal of water from oil/water mixtures.

\section{Experimental}

Porous nanofur membranes are fabricated using a hot pulling technique followed by pore perforation (Fig. 1a). Various thermoplastic polymers can be used as a base material for membrane fabrication, including biodegradable and shapememory polymers. ${ }^{4-50}$ To fabricate polycarbonate (PC) nanofur membrane, PC (Makrolon LED2045, Bayer, Germany) films with a thickness of $200 \mu \mathrm{m}$ were first fixed to the bottom plate of a hot embossing machine using a cyclic-olefin-copolymer (COC) sacrificial layer. ${ }^{49} \mathrm{~A}$ steel plate, roughened by sandblasting with rough aluminum silicate abrasive and aluminum oxide sand, serves as mold. During the hot pulling process, the plate is heated to $T \approx 215{ }^{\circ} \mathrm{C}$ and pressed into the PC film. Retraction of the heated plate results in elongation of the softened polymer and formation of a cratered polymer surface covered with high aspect ratio micro- and nanohairs. ${ }^{47-51}$ After removing the sacrificial COC film, nanofur is perforated by manually punching pores with a microneedle stamp (Edvers, Germany, 32 microneedles) or microneedles with different diameters (100 $\mu \mathrm{m}, 120 \mu \mathrm{m}$ (SEIRIN Co. Ltd., Japan), $160 \mu \mathrm{m}$ (Phoenix Medical Ltd., United Kingdom), $450 \mu \mathrm{m}$ (B. Braun Melsungen AG, Germany)). SEM images of the porous nanofur membrane are presented in Fig. $1 \mathrm{~b}$ and $\mathrm{c}$.

The nanofur surface is superhydrophobic with typical water contact angle WCA $>150^{\circ}$ (Fig. 2a) and superoleophilic with OCA close to $0^{\circ} .^{47}$ Due to these wetting properties, membranes fabricated from as-prepared nanofur are oil-removing. In order to fabricate water-removing membranes, the wetting properties of as-prepared membranes are inverted to underwater superoleophobic by argon plasma treatment, which is performed using a reactive ion etch system (Sentech $\mathrm{GmbH}$, Germany) at $0.2 \mathrm{mbar}$ and $30 \mathrm{~W}$ for 120 seconds. Plasma treated nanofur (pnanofur) is stored in water to ensure the long-term stability of its wetting properties by preventing hydrophobic recovery ${ }^{47}$

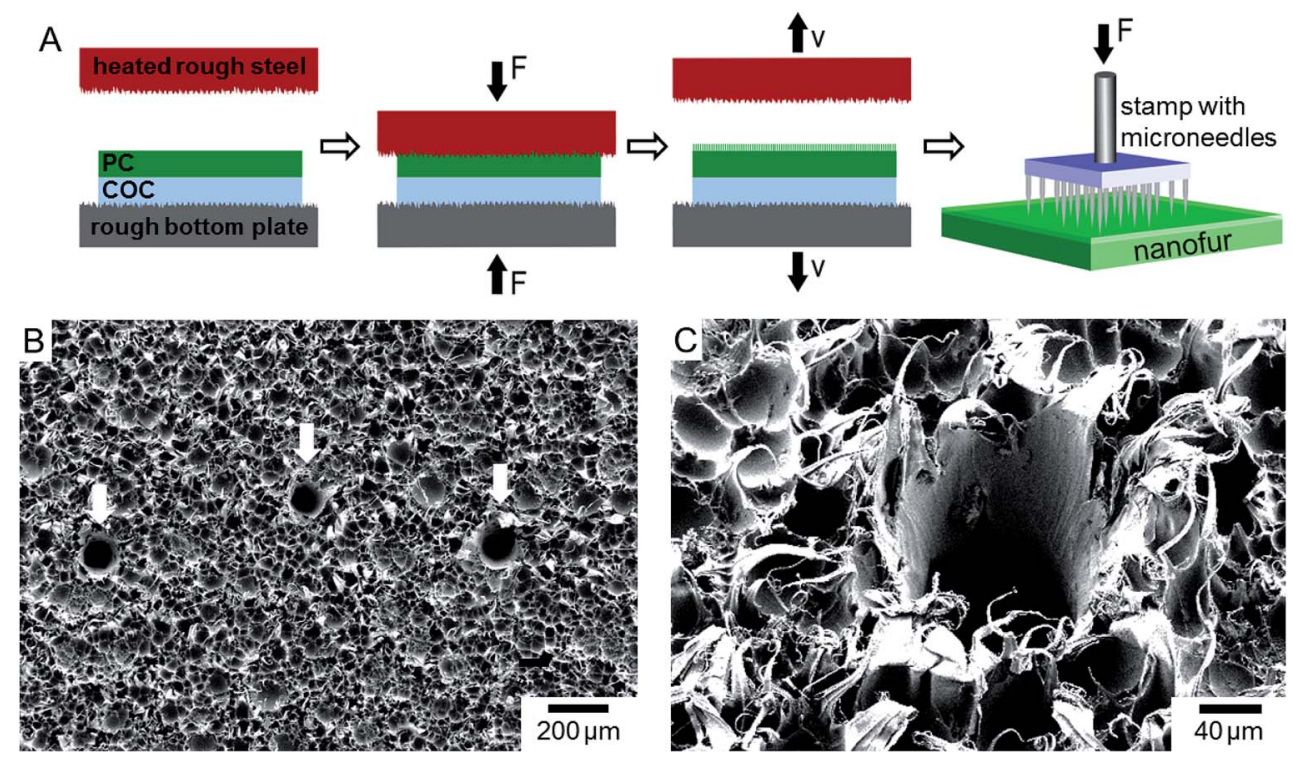

Fig. 1 (A) Schematic of the nanofur membrane fabrication process. Polycarbonate (PC) film is fixed to the bottom plate using a cyclic-olefincopolymer (COC) as a sacrificial layer. Next, a sandblasted steel plate is heated and pressed into PC film. Softened PC penetrates into the roughness of the heated plate and, after plate retraction, micro- and nanohairs are pulled from the film surface. Nanofur is perforated with a microneedle stamp to create a porous membrane. (B) SEM image of the porous nanofur membrane and (C) detailed view of a single pore. The average pore diameter is $80 \pm 25 \mu \mathrm{m}$. 
A

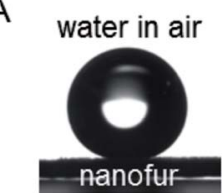

B

C

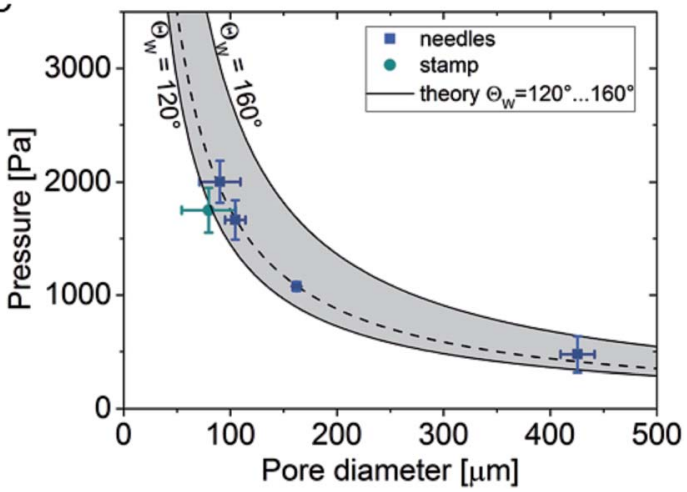

Fig. 2 Wetting behavior and water breakthrough pressure $\left(\Delta P_{B}\right)$ of superhydrophobic oil-removing nanofur membrane in air. (A) Water droplet on nanofur surface with water contact angle WCA $\approx 162^{\circ}$. (B) Schematic of $\Delta P_{\mathrm{B}}$ measurements on porous nanofur. (C) Experimental $\Delta P_{\mathrm{B}}$ as a function of pore diameter. Pores with different diameters were fabricated using microneedles and a microstamp. Theoretical $\Delta P_{\mathrm{B}}$ for WCA ranging from $120^{\circ}$ to $160^{\circ}$ is indicated by shaded area The fitted curve (dashed line) corresponds to WCA $\theta_{\mathrm{W}}=127^{\circ}$. As predicted by theory smaller pore sizes result in higher $\Delta P_{\mathrm{B}}$.

\section{Results and discussion}

\subsection{Oil-removing membrane}

High water breakthrough pressure $\Delta P_{\mathrm{B}}$ is a crucial parameter for efficient large volume separation of oil from oil/water mixtures using oil-removing membranes. $\Delta P_{\mathrm{B}}$ indicates the maximum water column height which can be retained by the membrane. ${ }^{9}$ To evaluate the ability of porous superhydrophobic nanofur to retain water, and study the influence of pore diameter on $\Delta P_{\mathrm{B}}$, we measured the breakthrough pressure of nanofur membranes with different pore sizes. The porous membrane was sealed between two vertical tubes and pressure was applied to its surface by continuously increasing the height of a water column (Fig. 2b). The breakthrough pressure was calculated from $\Delta P_{\mathrm{B}}=\rho g h_{\max }$, where $\rho$ is density of water, $g$ is gravitational acceleration and $h_{\max }$ is maximum height of the water column the membrane can support. ${ }^{10} h_{\max }$ was measured for membranes perforated with microneedles with different diameters $(100 \mu \mathrm{m}, 120 \mu \mathrm{m}, 160 \mu \mathrm{m}, 450 \mu \mathrm{m})$ and the microneedle stamp. The resulting pore diameters were determined from SEM images using ImageJ software. ${ }^{52}$ Experimental results shown in Fig. $2 \mathrm{c}$ demonstrate the influence of pore diameter on $\Delta P_{\mathrm{B}}$. As predicted by the theory, smaller pore diameters result in higher breakthrough pressures. For example, nanofur with $90 \pm$ $19 \mu \mathrm{m}$ pores retains water pressure of $2000 \pm 186 \mathrm{~Pa}(n=4)$, whereas pores with $426 \pm 16 \mu \mathrm{m}$ diameter retain only $477 \pm 158$ $\mathrm{Pa}(n=4)$. The average diameter of pores fabricated with the microneedle stamp is $80 \pm 25 \mu \mathrm{m}$ and $\Delta P_{\mathrm{B}}$ of these membranes is $1750 \pm 197 \mathrm{~Pa}$.
Next, experimentally measured breakthrough pressures of porous nanofur membranes are compared to theoretical $\Delta P_{\mathrm{B}}$ of hydrophobic membranes with cylindrical pores. $\Delta P_{\mathrm{B}}$ is calculated using Laplace-Young equation, ${ }^{10,15,53-55}$

$$
\Delta P_{\mathrm{B}}=\frac{2 \gamma\left|\cos \left(\theta_{\mathrm{W}}\right)\right|}{r},
$$

where $\theta_{\mathrm{W}}$ is WCA of the membrane surface, $\gamma_{\text {water }}=72.8 \mathrm{mN}$ $\mathrm{m}^{-1}$ is the water surface tension of water-air interface. ${ }^{56} \mathrm{As}$ can be seen in Fig. 2c, the experimental measurements are in good agreement with the theory. Fitting the experimental $\Delta P_{\mathrm{B}}$ values into eqn (1) results in WCA $\theta_{\mathrm{W}}=127^{\circ}$. The difference between this value and the measured WCA can be attributed to the variation in pore diameters and damage of nano- and microhairs at the pore edges. These results confirm that breakthrough pressure of nanofur membranes depend on pore diameters. Limitations for separating oil from oil/water mixtures in practical applications imposed by insufficient breakthrough pressure can be overcome by accordingly adapting pore diameter. ${ }^{9}$

For effective oil/water filtration by oil-removing porous membrane, water must be retained by the membrane in air as well as in oil. ${ }^{57}$ Superhydrophobic/superoleophilic properties of nanofur lead to oil being absorbed and retained in micro- and nanostructures on the nanofur surface. Similarly to a water droplet on the nanofur surface in air, water droplets placed on nanofur submerged in oil are repelled with an underoil WCA of $\sim 150^{\circ}$ and a roll-off angle below $5^{\circ}$ (Fig. 3a). Such underoil superhydrophobicity ensures selective penetration of oil through pores and retention of water. $\Delta P_{\mathrm{B}}$ measured on the nanofur membrane fabricated with the microstamp and prewetted with $n$-hexane is $\Delta P_{\mathrm{B}, \text { water/hexane }}=754 \pm 66 \mathrm{~Pa}$. According to eqn (1), the lower value of $\Delta P_{\mathrm{B}}$ of the membrane prewetted with hexane compared to the non-prewetted membrane is due to lower surface tension of the water/hexane

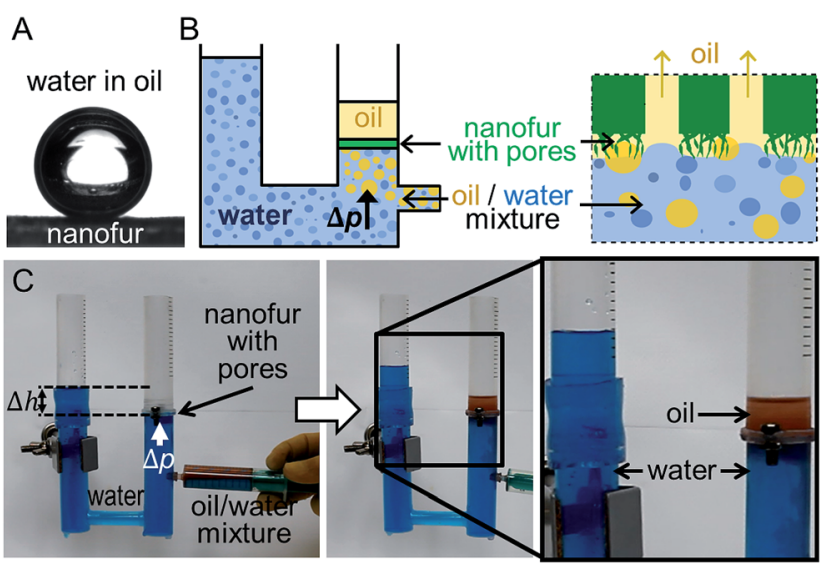

Fig. 3 Oil/water separation using oil-removing nanofur membrane. (A) Nanofur in oil is superhydrophobic with an underoil WCA $\approx 150^{\circ}$. (B) Schematic of the oil/water separation setup. (C) The membrane is fixed in the right tube with nanofur surface facing down. Pressure is applied through the water level. Hexane/water mixture is added under the membrane using a syringe. Oil penetrates through the pores, while water is retained. No water is observed in the collected oil. 
interface $\left(\gamma_{\text {water/hexane }}=51 \mathrm{mN} \mathrm{m}^{-1}\right)$ and lower underoil WCA, compared to $\gamma$ of water/air interface and WCA in air.

To demonstrate oil/water separation with oil-removing nanofur, porous membrane perforated with the microstamp was used to separate a $n$-hexane/water mixture in a filtration process. To overcome the limitation imposed by the lower density of hexane compared to water, we built an experimental setup in which the mixture is applied below the membrane (Fig. 3b and c). It consists of a U-shaped tube in which the nanofur faces downwards. The pipe is filled with water and water column height is used to pressurize the membrane with

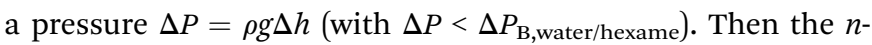
hexane/water mixture $(1: 1(\mathrm{v} / \mathrm{v}))$ is added under the membrane using a syringe (Fig. 3c). Due to its lower density, oil floats to the top and penetrates through the pores, while water is retained by the nanofur. The liquid flow stops when all oil is filtered through the nanofur, and no water is observed in the collected oil (Fig. 3c). Oil-removing membrane can be reused after washing it with isopropanol, which restores its wetting properties and oil absorption capacity. ${ }^{47}$ The same quality of oil/ water separation was achieved after reusing it four times.

\subsection{Water-removing membrane}

To separate water from the oil/water mixture, we fabricated a water-removing p-nanofur membrane by argon plasma treatment of the as-prepared nanofur membrane. Comparison of SEM images of the nanofur before and after argon plasma

\section{A}

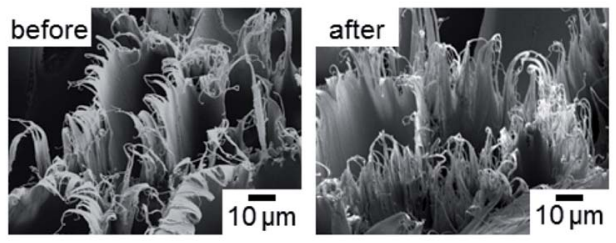

B

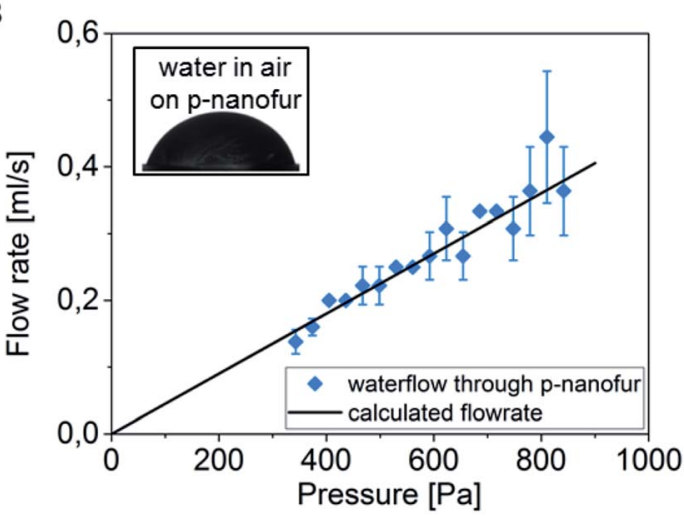

Fig. 4 Surface topography and properties of the plasma treated nanofur ( $p$-nanofur). (A) SEM images of nanofur before and after plasma treatment (arbitrarily chosen areas of the same sample) reveal no changes in surface topography. (B) Demonstration of wetting behaviour (inset: water droplet on $p$-nanofur with WCA $\approx 82^{\circ}$ ) and water permeability of $p$-nanofur. The water flow rate through hydrophilic porous $\mathrm{p}$-nanofur with $83 \pm 17 \mu \mathrm{m}$ pore diameter and $168 \mu \mathrm{m}$ pore length is in agreement with the flow rate calculated with eqn (2) ( $p=64$ micropores, $r=41.5 \mu \mathrm{m}, t_{\mathrm{m}}=168 \mu \mathrm{m}$ ). treatment did not reveal any changes in surface topography (see Fig. 4a). Plasma treatment leads to incorporation of hydrophilic carboxyl groups into the nanofur surface. The resulting increase in surface energy of the polycarbonate surface causes reduced WCA and underwater superoleophobicity. ${ }^{47,49}$ High water permeability is an important parameter for efficient waterremoving membranes. To characterize the water permeability of porous p-nanofur membranes, we measured water flow rates through the membranes sealed between two tubes. The porous p-nanofur membranes fabricated using the microstamp were used in this experiment $(n=4 ; 64$ micropores with average pore diameter $83 \pm 17 \mu \mathrm{m}$ and pore length $\left.t_{\mathrm{m}} \approx 168 \mu \mathrm{m}\right)$. The measured water flow rate linearly depends on the pressure, with lower pressure resulting in lower flow rate. In Fig. 4b, experimental water flow rates are compared to theoretical flow rates calculated from

$$
Q=\frac{\pi r^{4} p \Delta P}{8 \eta t_{\mathrm{m}}}
$$

where $r$ is pore radius, $p$ is number of pores, $\Delta P$ is applied pressure, $t_{\mathrm{m}}$ is pore length and $\eta$ is water viscosity. ${ }^{58}$ Experimental and theoretical results are in good agreement. Accordingly to the theory, membrane water flow rates can be further increased by increasing pore size, pressure or number of pores.

When p-nanofur is submerged under water, oil droplets are repelled by the underwater superoleophobic surface with an

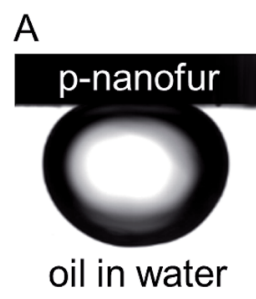

B
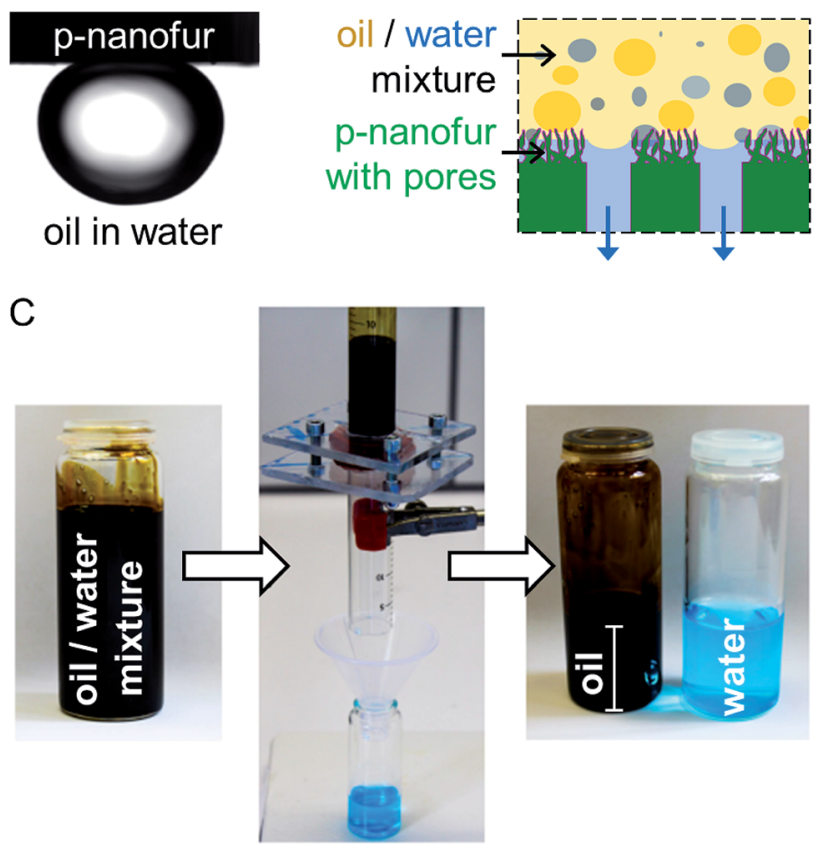

Fig. 5 Oil/water separation using the water-removing $p$-nanofur membrane. (A) $p$-Nanofur is underwater superoleophobic with $O C A \approx$ $152^{\circ}$. (B) Schematic of oil/water separation. Hydrophilic and underwater superoleophobic $\mathrm{p}$-nanofur allows water to penetrate through the pores and retains oil. (C) Mixture of crude oil and water (colored blue) is separated using a $p$-nanofur membrane. No crude oil is visible in the collected water. 
underwater OCA of $\sim 150^{\circ}$ and roll-off angle below $5^{\circ}$ (Fig. 5a). ${ }^{47,49}$ Underwater superoleophobicity of p-nanofur is a result of reduced contact between oil and solid due to water trapped in the surface micro- and nanostructures. ${ }^{59}$ To separate water from the oil/water mixture, water-removing porous $\mathrm{p}$ nanofur membrane retains the oil while allowing water to penetrate through, as schematically shown in Fig. 5b. To demonstrate this separation, a p-nanofur membrane was sealed between two tubes, and a mixture of crude oil (MiRO OK 679, Mineralölraffinerie Oberrhein, Germany) and water $(1: 1(\mathrm{v} / \mathrm{v}))$ is poured on the membrane. Water permeates through the porous p-nanofur membrane while oil is retained above (see Fig. 5c). No visible crude oil is observed in the collected water, demonstrating the high separation capability of the waterremoving porous p-nanofur membrane. Due to permeation of water and reduced oil/membrane contact, the p-nanofur membrane is not contaminated by crude oil and can be reused several times for this process. The same separation results were observed after reusing porous p-nanofur membrane up to four times.

\section{Conclusion}

We demonstrated selective separation of both oil and water from oil/water mixtures with porous nanofur membranes. Superhydrophobic oil-removing nanofur membranes are fabricated using the hot pulling technique followed by perforation with microneedles. Underwater superoleophobic waterremoving p-nanofur membranes were produced by additional argon plasma treatment of the as-prepared nanofur membranes. Nanofur is one of the few membrane materials which allows selective separation of both oil and water from oil/ water mixtures, whereas most membrane materials separate only one of the two liquids. Therefore, porous nanofur membranes can be used both for oil purification processes and in wastewater treatment technology. The control over filtration fluid can also help to overcome separation efficiency limitations imposed by such oil properties as viscosity and density, and to reduce the membrane fouling by oil. Moreover, we have shown that smaller pore sizes lead to higher breakthrough pressures, but decrease the flow rate. The presented fabrication technique, in which pores are fabricated in the nanofur films by perforation with microneedles, allows the selection of the pore diameter and their number accordingly to the specific requirements of the targeted separation process. Excellent performance, reusability and adaptable separation properties make porous nanofur membranes a promising material for large scale industrial and environmental oil/water filtration applications.

\section{Acknowledgements}

The authors thank Paul Abaffy, Uwe Köhler, Marc Schneider and Richard Thelen for their support with fabrication and characterization. C. Z. acknowledges a scholarship from Carl Zeiss Foundation. F. V. acknowledges a scholarship from the Landesgraduiertenförderung Baden-Württemberg. Crude oil was kindly provided by MiRO Mineralölraffinerie Oberrhein
GmbH \& Co. KG. We acknowledge support by Deutsche Forschungsgemeinschaft and Open Access Publishing Fund of Karlsruhe Institute of Technology. This work was partly carried out with the support of the Karlsruhe Nano Micro Facility (KNMF, http://www.kit.edu/knmf), a Helmholtz Research Infrastructure at Karlsruhe Institute of Technology (KIT, http:// www.kit.edu).

\section{References}

1 S. B. Joye, Science, 2015, 349, 592-593.

2 M. Schrope, Nature, 2011, 472, 152-154.

3 C. H. Lee, B. Tiwari, D. Zhang and Y. K. Yap, Environ. Sci.: Nano, 2017, 4, 514-525.

4 D. Fragouli and A. Athanassiou, Nat. Nanotechnol., 2017, 12, 406-407.

5 Z. Xue, Y. Cao, N. Liu, L. Feng and L. Jiang, J. Mater. Chem. A, 2014, 2, 2445-2460.

6 Q. Ma, C. Honfei, A. G. Fane, W. Rong and Z. Hua, Small, 2016, 12, 2189-2202.

7 M. Adebajo, R. Frost, J. Kloprogge, O. Carmody and S. Kokot, J. Porous Mater., 2003, 10, 159-170.

8 Z. Chu, Y. Feng and S. Seeger, Angew. Chem., Int. Ed., 2015, 54, 2328-2338.

9 P.-C. Chen and Z.-K. Xu, Sci. Rep., 2013, 3, 2776.

10 Z. Xue, S. Wang, L. Lin, L. Chen, M. Liu, L. Feng and L. Jiang, Adv. Mater., 2011, 23, 4270-4273.

11 L. Wen, Y. Tian and L. Jiang, Angew. Chem., Int. Ed., 2015, 54, 2-15.

12 C. Zeiger, I. C. Rodrigues da Silva, M. Mail, M. N. Kavalenka, W. Barthlott and H. Hölscher, Bioinspiration Biomimetics, 2016, 11, 056003.

13 B. M. Liu, S. Wang, Z. Wei, Y. Song and L. Jiang, Adv. Mater., 2009, 21, 665-669.

14 C. Guo, S. Wang, H. Liu, L. Feng, Y. Song and L. Jiang, J. Adhes. Sci. Technol., 2008, 22, 395-402.

15 A. Lafuma and D. Quéré, Nat. Mater., 2003, 2, 457-460.

16 J. Li, D. Li, Y. Yang, J. Li, F. Zha and Z. Lei, Green Chem., 2016, 18, 541-549.

17 J. Gu, P. Xiao, Y. Huang, J. Zhang and T. Chen, J. Mater. Chem. A, 2015, 3, 4124-4128.

18 J. Y. Huang, S. H. Li, M. Z. Ge, L. N. Wang, T. L. Xing, G. Q. Chen, X. F. Liu, S. S. Al-Deyab, K. Q. Zhang, T. Chen and Y. K. Lai, J. Mater. Chem. A, 2015, 3, 2825-2832.

19 F. Zhang, W. B. Zhang, Z. Shi, D. Wang, J. Jin and L. Jiang, Adv. Mater., 2013, 25, 4192-4198.

20 C.-H. Xue, Y.-R. Li, J.-L. Hou, L. Zhang, J.-Z. Ma and S.-T. Jia, J. Mater. Chem. A, 2015, 3, 10248-10253.

21 L.-H. Kong, X.-H. Chen, L.-G. Yu, Z.-S. Wu and P.-Y. Zhang, ACS Appl. Mater. Interfaces, 2015, 7, 2616-2625.

22 Z. Wang, Y. Xu, Y. Liu and L. Shao, J. Mater. Chem. A, 2015, 3, 12171-12178.

23 L. Feng, Z. Zhang, Z. Mai, Y. Ma, B. Liu, L. Jiang and D. Zhu, Angew. Chem., Int. Ed., 2004, 43, 2012-2014.

24 X. Chen, Y. He, Y. Fan, Q. Yang, G. Zeng and H. Shi, J. Mater. Sci., 2017, 52, 2169-2179. 
25 C. H. Lee, N. Johnson, J. Drelich and Y. K. Yap, Carbon, 2011, 49, 669-676.

26 C. Xiao, L. Si, Y. Liu, G. Guan, D. Wu, Z. Wang and X. Hao, J. Mater. Chem. A, 2016, 4, 8080-8090.

27 W. Zhang, N. Liu, Y. Cao, Y. Chen, L. Xu, X. Lin and L. Feng, Adv. Mater., 2015, 27, 7349-7355.

28 L. Li, Z. Liu, Q. Zhang, C. Meng, T. Zhang and J. Zhai, J. Mater. Chem. A, 2015, 3, 1279-1286.

29 J. Li, L. Yan, H. Li, W. Li, F. Zha and Z. Lei, J. Mater. Chem. A, 2015, 3, 14696-14702.

30 C.-F. Wang, F.-S. Tzeng, H.-G. Chen and C.-J. Chang, Langmuir, 2012, 28, 10015-10019.

31 Q. Wen, J. Di, L. Jiang, J. Yu and R. Xu, Chem. Sci., 2013, 4, 591-595.

32 X. Zheng, Z. Guo, D. Tian, X. Zhang, W. Li and L. Jiang, ACS Appl. Mater. Interfaces, 2015, 7, 4336-4343.

33 C. Du, J. Wang, Z. Chen and D. Chen, Appl. Surf. Sci., 2014, 313, 304-310.

34 S. J. Maguire-Boyle and A. R. Barron, J. Membr. Sci., 2011, 382, 107-115.

35 K. He, H. Duan, G. Y. Chen, X. Liu, W. Yang and D. Wang, ACS Nano, 2015, 9, 9188-9198.

36 Z. He, X. Zhang and W. Batchelor, RSC Adv., 2016, 6, 2143521438.

37 L. Zhang, Y. Zhong, D. Cha and P. Wang, Sci. Rep., 2013, 3, 15.

38 J. Yang, Z. Zhang, X. Xu, X. Zhu, X. Men and X. Zhou, J. Mater. Chem., 2012, 22, 2834-2837.

39 J.-B. Fan, Y. Song, S. Wang, J. Meng, G. Yang, X. Guo, L. Feng and L. Jiang, Adv. Funct. Mater., 2015, 25, 5368-5375.

40 G. Kwon, A. K. Kota, Y. Li, A. Sohani, J. M. Mabry and A. Tuteja, Adv. Mater., 2012, 24, 3666-3671.

41 J. Wu, N. Wang, Y. Zhao and L. Jiang, Nanoscale, 2015, 7, 2625-2632.

42 R. Ou, J. Wei, L. Jiang, G. P. Simon and H. Wang, âĂŐEnviron. Sci. Technol., 2016, 50, 906-914.

43 Z. Cheng, J. Wang, H. Lai, Y. Du, R. Hou, C. Li, N. Zhang and K. Sun, Langmuir, 2015, 31, 1393-1399.
44 M. Tao, L. Xue, F. Liu and L. Jiang, Adv. Mater., 2014, 26, 2943-2948.

45 X. Du, S. You, X. Wang, Q. Wang and J. Lu, Chem. Eng. J., 2017, 313, 398-403.

46 D. Ge, L. Yang, C. Wang, E. Lee, Y. Zhang and S. Yang, Chem. Commun., 2015, 51, 6149-6152.

47 M. N. Kavalenka, F. Vüllers, J. Kumberg, C. Zeiger, V. Trouillet, S. Stein, T. T. Ava, C. Li, M. Worgull and H. Hölscher, Sci. Rep., 2017, 7, 39970.

48 M. Röhrig, M. Mail, M. Schneider, H. Louvin, A. Hopf, T. Schimmel, M. Worgull and H. Hölscher, Adv. Mater. Interfaces, 2014, 1, 1300083.

49 F. Vüllers, G. Gomard, J. B. Preinfalk, E. Klampaftis, M. Worgull, B. Richards, H. Hölscher and M. N. Kavalenka, Small, 2016, 12, 6144-6152.

50 M. N. Kavalenka, F. Vüllers, S. Lischker, C. Zeiger, A. Hopf, M. Röhrig, B. E. Rapp, M. Worgull and H. Hölscher, ACS Appl. Mater. Interfaces, 2015, 7, 10651-10655.

51 M. N. Kavalenka, A. Hopf, M. Schneider, M. Worgull and H. Holscher, RSC Adv., 2014, 4, 31079-31083.

52 ImageJ Software, imagej.net.

53 M. A. Gondal, M. S. Sadullah, M. A. Dastageer, G. H. McKinley, D. Panchanathan and K. K. Varanasi, ACS Appl. Mater. Interfaces, 2014, 6, 13422-13429.

54 A. M. Vadya, P. J. Halling and G. Bell, Biotechnol. Bioeng., 1994, 44, 765-571.

55 J. W. Grate, K. J. Dehoff, M. G. Warner, J. W. Pittman, T. W. Wietsma, C. Zhang and M. Oostrom, Langmuir, 2012, 28, 7182-7188.

56 G. M. Kontogeorgis and G. K. Folas, in Thermodynamics and Colloid and Surface Chemistry, John Wiley \& Sons, Ltd, 2009, pp. 577-611.

57 Y. Lu, S. Sathasivam, J. Song, C. R. Crick, C. J. Carmalt and I. P. Parkin, Science, 2015, 347, 1132-1135.

58 G. K. Batchelor, An Introduction in Fluid Dynamics, Cambridge University Press, Cambridge, 1970, p. 180.

59 J. Yong, F. Chen, Q. Yang, H. Bian, G. Du, C. Shan, J. Huo, Y. Fang and X. Hou, Adv. Mater. Interfaces, 2016, 3, 1500650. 